\title{
A semicontinuous trace for almost local operators on an open manifold
}

\author{
Daniele Guido ${ }^{1}$, Tommaso Isola ${ }^{2}$ \\ (1) Dipartimento di Matematica, \\ Università della Basilicata, \\ I-85100 Potenza, Italy. \\ (2) Dipartimento di Matematica, \\ Università di Roma "Tor Vergata", \\ I-00133 Roma, Italy. \\ guido@unibas.it, isola@mat.uniroma2.it
}

April 23, 2001

\begin{abstract}
A semicontinuous semifinite trace is constructed on the $\mathrm{C}^{*}$-algebra generated by the finite propagation operators acting on the $\mathrm{L}^{2}$-sections of a hermitian vector bundle on an amenable open manifold of bounded geometry. This trace is the semicontinuous regularization of a functional already considered by J. Roe. As an application, we show that, by means of this semicontinuous trace, Novikov-Shubin numbers for amenable manifolds can be defined.
\end{abstract}




\section{Introduction.}

In this paper, we construct a $\mathrm{C}^{*}$-algebra of operators on the Hilbert space of $L^{2}$-sections of any vector bundle on an open manifold with bounded geometry, together with a semicontinuous semifinite trace on it which is finite on locally trace-class operators with suitable uniform bounds. In some sense our $\mathrm{C}^{*}$ algebra will be maximal with respect to these properties.

The finite-dimensionality of the eigenspaces of elliptic differential operators on a compact manifold is one of the classical results allowing us to define of many analytical quantities which then turn out to have a geometric meaning. The possibility of defining analogous invariants for open manifolds is related to the possibility of renormalizing the dimension of such spaces, in order to give a new sense to finiteness.

Since the dimension of a space can be defined as the trace of its orthogonal projection, one can try to perform the renormalization by replacing the usual trace with another one. As eigenprojections of elliptic differential operators on an open manifold are not compact in general, no trace on $B(H)$ can be finite on them, so the required trace can only be defined on a sub-algebra.

The first step in this direction is due to Atiyah in the seminal paper on the index theorem for covering manifolds [1]. He observed that, when the manifold has the structure of an infinite covering of a compact manifold with respect to a group $\Gamma, \Gamma$-periodic operators form a type $\mathrm{II}_{\infty}$ von Neumann algebra. If the size of the eigenprojections of $\Gamma$-periodic elliptic differential operators is measured with the normal trace on the von Neumann algebra of $\Gamma$-periodic operators, the classical finiteness is recovered. This started the whole theory of $\mathrm{L}^{2}$-invariants, producing both new versions of classical invariants, such as $\mathrm{L}^{2}$-Betti numbers, and completely new objects, such as Novikov-Shubin numbers.

The idea of extending Atiyah's construction, without any reference to a group, is due to John Roe [19] and is based on the following procedure: choose a suitable invasion of the manifold via compact sets (amenable exhaustion), normalize the trace on such sets dividing by the measure of the set, go to infinity by a suitable generalized limit. Applying this procedure to finite propagation operators gives a trace.

In order to produce a semicontinuous semifinite trace on a $\mathrm{C}^{*}$-algebra containing finite propagation operators, some technical problems have to be solved, and this is the purpose of this paper. Thus, we choose as a $\mathrm{C}^{*}$-algebra simply the norm closure of the finite propagation operators, in some sense a maximal choice if one requires the trace property. Further we observe that with the naive definition of the functional is not semicontinuous and has a weak trace property, so we regularize to obtain a semicontinuous semifinite positive trace on the $\mathrm{C}^{*}$-algebra.

While the $\mathrm{C}^{*}$-algebra only depends on the metric on the manifold $M$, the trace $T r_{\mathcal{K}, \omega}$ depends upon the large scale geometry of $M$, described by the amenable exhaustion $\mathcal{K}$, and on a generalized limit procedure $\omega$. We expect geometrically interesting quantities derived from $T r_{\mathcal{K}, \omega}$ to be independent of $\omega$.

The domain of the trace $\mathcal{D}\left(\operatorname{Tr}_{\mathcal{K}, \omega}\right)$ contains all compact operators, and in- 
deeed $\operatorname{Tr} r_{\mathcal{K}, \omega}$ vanishes on them, for any $\omega$. Moreover $\mathcal{D}\left(\operatorname{Tr}_{\mathcal{K}, \omega}\right)$ contains integral operators with off-diagonal exponentially decaying kernels, and, if the kernel is also uniformly continuous on the diagonal, the trace of the integral operator can be computed using the simple procedure devised by J. Roe.

As $C_{c}$-functional calculi of $\Delta_{p}$ belong to $\mathcal{D}\left(T r_{\mathcal{K}, \omega}\right)$, the spectral density function $N_{p}$ of $\Delta_{p}$ is defined. Indeed, as $f \in C_{c}[0, \infty) \rightarrow \operatorname{Tr}_{\mathcal{K}, \omega}\left(f\left(\Delta_{p}\right)\right) \in \mathbb{C}$ is a positive linear functional, it defines a Radon measure $\mu_{p}$ on $[0, \infty)$, hence we set $N_{p}(t):=\mu_{p}((0, t))$.

As an application, one can define, in analogy with the case of covering manifolds [1] 17], both the $\mathrm{L}^{2}$-Betti numbers and the Novikov-Shubin numbers of $M$. Note that $\mathrm{L}^{2}$-Betti numbers were also defined (differently but equivalently) by J. Roe [20]. We prove here that, for open manifolds of bounded geometry, the 0th Novikov-Shubin number is always $\geq 1$ thus extending a result of Varopoulos [24.

As it is known, a general understanding of the geometric meaning of the Novikov-Shubin invariants is still lacking. We believe that some aspects can be better understood by interpreting them as global invariants of an open manifold, rather than as homotopy invariants of a compact one.

The asymptotic character of these numbers manifests itself at two points in their construction. On the one hand, the trace used to define these numbers is a large scale trace, since, as observed by Roe [19], it is given by an average over the group, in the case of coverings, and by an average on the exhaustion, in the case of open manifolds. On the other hand, these numbers are defined in terms of the low frequency behaviour of the $p$-Laplacians, or the large time behaviour of the $p$-heat kernel. In this respect, they are the large scale counterpart of the spectral dimension, namely of the dimension as defined by the Weyl asymptotics.

On the technical side, the functional $\varphi$ considered by J. Roe has two main problems. The first is that it is not semicontinuous; in fact its kernel is not closed (see Proposition 4.1); the second is that the equality $\varphi(a b)=\varphi(b a)$ has only been proved for $a, b$ uniformly smoothing operators of order $-\infty$ in the domain of $\varphi$. Conversely, the trace property on a $\mathrm{C}^{*}$-algebra requires the equality $\varphi(a b)=\varphi(b a)$ to hold for $a$ in the domain and $b$ in the $\mathrm{C}^{*}$-algebra. This is proved for the regularized trace in Theorem 4.2 .

These two properties play a key role in showing that Novikov-Shubin numbers are invariant under quasi-isometries and that they are asymptotic dimensions in the sense of noncommutative geometry, which is done in [11.

Some of the results contained in the present paper have been announced in several international conferences. In particular we would like to thank the Erwin Schrödinger Institute in Vienna, where a preliminary version of this paper was completed, and the organisers of the "Spectral Geometry Program" for their kind invitation.

\section{Open manifolds of bounded geometry}




\subsection{Preliminaries}

In this subsection we give some preliminary results on open manifolds of bounded geometry that are needed in the sequel.

Several definitions of bounded geometry for an open manifold (i.e. a noncompact complete Riemannian manifold) are usually considered. They all require some uniform bound (either from above or from below) on some geometric objects, such as: injectivity radius, sectional curvature, Ricci curvature, Riemann curvature tensor etc. (For all unexplained notions see e.g. Chavel's book [3]).

In this paper the following form is used, but see [4] and references therein for a different approach.

Definition 1.1. Let $(M, g)$ be a complete Riemannian manifold. We say that $M$ has $\mathrm{C}^{\infty}$-bounded geometry if it has positive injectivity radius, and the curvature tensor is bounded, together with all its covariant derivatives.

Lemma 1.2. Let $M$ be an $n$-dimensional complete Riemannian manifold with positive injectivity radius, sectional curvature bounded from above, and Ricci curvature bounded from below, in particular $M$ could have $C^{\infty}$-bounded geometry. Then there are real functions $\beta_{1}$, $\beta_{2}$ s.t.

(i) for all $x \in M, r>0$,

$$
0<\beta_{1}(r) \leq \operatorname{vol}(B(x, r)) \leq \beta_{2}(r)
$$

(ii) $\lim _{r \rightarrow 0} \frac{\beta_{2}(r)}{\beta_{1}(r)}=1$.

Proof. (i) We can assume, without loss of generality, that the sectional curvature is bounded from above by some positive constant $c_{1}$, and the Ricci curvature is bounded from below by $(n-1) c_{2} g$, with $c_{2}<0$. Then, denoting with $V_{\delta}(r)$ the volume of a ball of radius $r$ in a manifold of constant sectional curvature equal to $\delta$, we can set $\beta_{1}(r):=V_{c_{1}}\left(r \wedge r_{0}\right)$, and $\beta_{2}:=V_{c_{2}}(r)$, where $r_{0}:=$ $\min \left\{\operatorname{inj}(M), \frac{\pi}{\sqrt{c_{1}}}\right\}$, and $\operatorname{inj}(M)$ is the injectivity radius of $M$. Then the result follows from ([3], p.119,123).

(ii)

$$
\begin{aligned}
\lim _{r \rightarrow 0} \frac{\beta_{2}(r)}{\beta_{1}(r)} & =\lim _{r \rightarrow 0} \frac{V_{c_{2}}(r)}{V_{c_{1}}(r)}=\lim _{r \rightarrow 0} \frac{\int_{0}^{r} S_{c_{2}}(t)^{n-1} d t}{\int_{0}^{r} S_{c_{1}}(t)^{n-1} d t} \\
& =\left(\lim _{r \rightarrow 0} \frac{S_{c_{2}}(r)}{S_{c_{1}}(r)}\right)^{n-1}=1
\end{aligned}
$$

where (cfr. [3], formulas (2.48), (3.24), (3.25)) $V_{\delta}(r)=\frac{n \sqrt{\pi}}{\Gamma(n / 2+1)} \int_{0}^{r} S_{\delta}(t)^{n-1} d t$, and

$$
S_{\delta}(r):= \begin{cases}\frac{1}{\sqrt{-\delta}} \sinh (r \sqrt{-\delta}) & \delta<0 \\ r & \delta=0 \\ \frac{1}{\sqrt{\delta}} \sin (r \sqrt{\delta}) & \delta>0 .\end{cases}
$$


Let $M$ be a complete Riemannian manifold, and recall ([26]) that $\Delta_{p}:=(d+$ $\left.d^{*}\right)\left.^{2}\right|_{L^{2}\left(\Lambda^{p} T^{*} M\right)}$, the $p$-th Laplacian on $M$, is essentially self-adjoint and positive, and the semigroup $\mathrm{e}^{-t \Delta_{p}}$ has a $\mathrm{C}^{\infty}$ kernel, $H_{p}(t, x, y)$, on $(0, \infty) \times M \times M$, called the $p$-th heat kernel. Let us mention the following result, which will be useful in the sequel.

Proposition 1.3. Let $M$ be an n-dimensional complete Riemannian manifold with $C^{\infty}$-bounded geometry, then for all $T>0$, there are $c, c^{\prime}>0$, s.t., for $0<t \leq T$,

$$
\begin{gathered}
\left|H_{p}(t, x, y)\right| \leq c t^{-n / 2-1} \exp \left(\frac{-c^{\prime} \delta(x, y)^{2}}{t}\right) \\
\left|\nabla_{x} H_{p}(t, x, y)\right| \leq c t^{-n / 2-3 / 2} \exp \left(\frac{-c^{\prime} \delta(x, y)^{2}}{t}\right)
\end{gathered}
$$

where we denoted with $\delta$ the metric induced on $M$ by $g$. As a consequence $H_{p}(t, \cdot, \cdot)$ is uniformly continuous on a neighborhood of the diagonal of $M \times M$.

Proof. The estimates are proved in [2]. For the last statement, for any $\delta_{0}<$ $\min \left\{1, \operatorname{inj}(M), \frac{\pi}{\sqrt{c_{1}}}\right\}, x \in M, y \in B\left(x, \delta_{0}\right)$, we have $\left|H_{p}(t, x, y)-H_{p}(t, x, x)\right| \leq$ $\sup \left|\nabla_{y} H_{p}(t, x, y)\right| \delta(x, y)$, and we get the uniform continuity.

\subsection{The $\mathrm{C}^{*}$-algebra of almost local operators}

Here we introduce the $\mathrm{C}^{*}$-algebra of almost local operators, and observe that the $p$-heat semigroup belongs to it.

Let $F$ be a finite dimensional Hermitian vector bundle over $M$, and let $L^{2}(F)$ be the Hilbert space completion of the smooth sections with compact support of $F$ w.r.t. the scalar product $\left\langle s_{1}, s_{2}\right\rangle:=\int_{M}\left\langle s_{1 x}, s_{2 x}\right\rangle d v o l(x)$. The following lemmawill beused in the sequel.

Lemma 1.4. Let $A$ be a bounded self-adjoint operator on $L^{2}(F)$, with measurable kernel. Then

$$
\|A\| \leq \sup _{x \in M} \int_{M}|a(x, y)| d y
$$

Proof. Since $A$ is self-adjoint, $a(x, y)$ is symmetric, hence

$$
\begin{aligned}
\|A\|_{1 \rightarrow 1} & =\sup \left\{|(f, A g)|: f \in L^{\infty}(F),\|f\|_{\infty}=1, g \in L^{1}(F),\|g\|_{1}=1\right\} \\
& \leq \sup _{x \in M} \int_{M}|a(y, x)| d y=\|A\|_{\infty \rightarrow \infty}
\end{aligned}
$$

The thesis easily follows from Riesz-Thorin interpolation theorem.

Recall [21] that an operator $A \in \mathcal{B}\left(L^{2}(F)\right)$ has finite propagation if there is a constant $u_{A}>0$ s.t. for any compact subset $K$ of $M$, any $\varphi \in L^{2}(F)$, $\operatorname{supp} \varphi \subset K$, we have $\operatorname{supp} A \varphi \subset P e n^{+}\left(K, u_{A}\right):=\left\{x \in M: \delta(x, K) \leq u_{A}\right\}$. Clearly finite propagation operators for a ${ }^{*}$-subalgebra of $\mathcal{B}\left(L^{2}(F)\right)$, denoted by $\mathcal{A}_{0} \equiv \mathcal{A}_{0}(F)$. 
Definition 1.5. We call the norm closure of $\mathcal{A}_{0}$ the $\mathrm{C}^{*}$-algebra of almost local operators on $L^{2}(F)$, and denote it by $\mathcal{A} \equiv \mathcal{A}(F)$.

Theorem 1.6. Let $M$ be a complete Riemannian manifold of $C^{\infty}$-bounded geometry. Then the $C^{*}$-algebra of almost local operators on $L^{2}\left(\Lambda^{p} T^{*} M\right)$ contains all compact operators and the $C_{0}([0, \infty))$-functional calculus of the Laplace operator on $p$-forms, i.e. $f\left(\Delta_{p}\right) \in \mathcal{A}\left(\Lambda^{p} T^{*} M\right)$, for any $f \in C_{0}([0, \infty))$

Proof. Indeed J. Roe shows that compact operators (21], Lemma 4.12) and $C_{0}([0, \infty))$-functional calculi of $\Delta_{p}\left([22]\right.$, Proposition 3.6) belong to the $\mathrm{C}^{*}$ algebra $C^{*}(M)$, which consists of the locally compact operators in $\mathcal{A}$.

\section{A functional described by J. Roe}

This section is devoted to the construction of a trace on the $\mathrm{C}^{*}$-algebra $\mathcal{A}=$ $\mathcal{A}(F)$. The basic idea for this construction is due to Roe [19], and is based on a regular exhaustion for the manifold. We shall regularize this functional, in order to get a semicontinuous semifinite trace on the $\mathrm{C}^{*}$-algebra of almost local operators. As observed by Roe, this trace is strictly related to the trace constructed by Atiyah [1] in the case of covering manifolds. It may therefore be used to define the Novikov-Shubin invariants for open manifolds, as we do in section 1 .

In the rest of this paper $M$ is a complete Riemannian manifold of $\mathrm{C}^{\infty}$ bounded geometry as in Definition 1.1, that we assume endowed with a regular exhaustion, as in the following Definition.

Definition 2.1. 19 A regular exhaustion $\mathcal{K}$ of $M$ is an increasing sequence $\left\{K_{n}\right\}$ of compact subsets of $M$, whose union is $M$, and s.t., for any $r>0$

$$
\lim _{n \rightarrow \infty} \frac{\operatorname{vol}\left(K_{n}(r)\right)}{\operatorname{vol}\left(K_{n}(-r)\right)}=1,
$$

where $K(r) \equiv \operatorname{Pen}^{+}(K, r):=\{x \in M: \delta(x, K) \leq r\}$, and $K(-r) \equiv P e n^{-}(K, r):=$ the closure of $M \backslash \mathrm{Pen}^{+}(M \backslash K, r)$.

Observe that, as $M$ is complete, $\mathrm{Pen}^{+}(K, r)$ coincides with the closure of $\{x \in M: \delta(x, K)<r\}$, which is the original definition of Roe.

Lemma 2.2. Let $K$ be a compact subset of $M$, then

(i) $K\left(-r_{2}\right) \subset K \subset K\left(r_{1}\right)$, for any $r_{1}, r_{2}>0$

(ii) $\{x \in M: \delta(x, M \backslash K)<r\} \subset$ Interior of $\operatorname{Pen}^{+}(M \backslash K, r) \equiv M \backslash K(-r)$

(iii) $\operatorname{Pen}^{+}\left(K\left(r_{1}\right) \backslash K\left(-r_{2}\right), R\right) \subset K\left(r_{1}+R+\varepsilon\right) \backslash K\left(-r_{2}-R-\varepsilon\right)$, for any $r_{1}, r_{2}, R, \varepsilon>0$.

Proof. (ii) If $\delta(x, M \backslash K)<r$, there is $z \in M \backslash K$ s.t. $\delta(x, z)<r$, so that $x$ belongs to the interior of $\mathrm{Pen}^{+}(M \backslash K, r)$, which is the complement of $K(-r)$. 
(iii) Indeed if $x \in \operatorname{Pen}^{+}\left(K\left(r_{1}\right) \backslash K\left(-r_{2}\right), R\right)$, then for any $\varepsilon>0$ there is $x_{\varepsilon} \in K\left(r_{1}\right) \backslash K\left(-r_{2}\right)$ with $\delta\left(x, x_{\varepsilon}\right)<R+\varepsilon / 2$. Therefore, on the one hand, $\delta(x, K) \leq R+\varepsilon / 2+r_{1}$, which implies $x \in K\left(r_{1}+R+\varepsilon\right)$. On the other hand, as $x_{\varepsilon} \notin K\left(-r_{2}\right)$, there is $y_{\varepsilon} \in M \backslash K$ s.t. $\delta\left(y_{\varepsilon}, x_{\varepsilon}\right)<r_{2}+\frac{\varepsilon}{2}$, hence $\delta\left(x, y_{\varepsilon}\right) \leq \frac{\varepsilon}{2}+R+r_{2}+\frac{\varepsilon}{2}$ and $x \notin K\left(-r_{2}-R-\varepsilon\right)$.

Following Moore-Schochet [16], we recall that an operator $T$ on $L^{2}(F)$ is called locally trace class if, for any compact set $K \subset M, E_{K} T E_{K}$ is trace class, where $E_{K}$ denotes the projection given by the characteristic function of $K$. It is known that the functional $\mu_{T}(K):=\operatorname{Tr}\left(E_{K} T E_{K}\right)$ extends to a Radon measure on $M$. To state the next definition we need some preliminary notions.

Definition 2.3. Define $\mathfrak{J}_{0+} \equiv \mathfrak{J}_{0+}(F)$ as the set of positive locally trace class operators $T$, such that

(i) there is $c>0$ s.t. $\mu_{T}\left(K_{n}\right) \leq c \operatorname{vol}\left(K_{n}\right)$, for all $n \in \mathbb{N}$,

(ii) $\lim _{n \rightarrow \infty} \frac{\mu_{T}\left(K_{n}\left(r_{1}\right) \backslash K_{n}\left(-r_{2}\right)\right)}{\operatorname{vol}\left(K_{n}\right)}=0$, for all $r_{1}, r_{2}>0$.

Lemma 2.4. $\mathcal{J}_{0+}$ is a hereditary (positive) cone in $\mathcal{B}\left(L^{2}(F)\right)$.

Proof. Linearity follows by $\mu_{A+B}=\mu_{A}+\mu_{B}$. If $T \in \mathcal{J}_{0+}$, and $0 \leq A \leq T$, then $\operatorname{Tr}\left(B A B^{*}\right) \leq \operatorname{Tr}\left(B T B^{*}\right)$, for any $B \in \mathcal{B}\left(L^{2}(F)\right)$, and the thesis follows.

Remark 2.5. The hereditary cone $\mathcal{J}_{0+}$ depends on the exhaustion $\mathcal{K}$, however it contains a (hereditary) subcone, given by the operators $T$ for which there is $c>0$ such that $\mu_{T}(\Omega) \leq c \operatorname{vol}(\Omega)$ for any measurable set $\Omega$. Proposition 1.3 implies that the operator $e^{-t \Delta_{p}}$ belongs to the subcone, hence to $\mathcal{J}_{0+}\left(\Lambda^{p} T^{*} M\right)$.

Recall [19] that $\mathcal{U}_{-\infty}(F)$ is the set of uniform operators of order $-\infty$.

Proposition 2.6. $\mathfrak{U}_{-\infty}(F)_{+} \subset \mathfrak{J}_{0+}(F)$.

Proof. Let $A \in \mathcal{U}_{-\infty}(F)$, so that $A u(x)=\int_{M} a(x, y) u(y) d y$, with $a \in C^{\infty}(F \otimes$ $F)$ is a smoothing kernel, and is uniformly bounded together with all its covariant derivatives $([19], 2.9)$. Then for any Borel set $\Omega \subset M, \mu_{A}(\Omega)=$ $\operatorname{Tr}\left(E_{\Omega} A E_{\Omega}\right)=\int_{\Omega} \operatorname{tr}(a(x, x)) d x \leq c \operatorname{vol}(\Omega)$, and the result easily follows.

If $\omega$ is a state on $\ell^{\infty}(\mathbb{N})$ vanishing on infinitesimal sequences, we use in the following the notation $\operatorname{Lim}_{\omega} a_{n}:=\omega\left(\left\{a_{n}\right\}\right)$, for any $\left\{a_{n}\right\} \in \ell^{\infty}(\mathbb{N})$. Consider the weight $\varphi \equiv \varphi_{\mathcal{K}, \omega}$ on $\mathcal{B}\left(L^{2}(F)\right)_{+}$given by

$$
\varphi(A):= \begin{cases}\operatorname{Lim}_{\omega} \frac{\mu_{A}\left(K_{n}\right)}{\operatorname{vol}\left(K_{n}\right)} & A \in \mathcal{J}_{0+} \\ +\infty & A \in \mathcal{B}\left(L^{2}(F)\right)_{+} \backslash \mathfrak{J}_{0+} .\end{cases}
$$

Observe that the functional $\varphi$ is the functional defined by Roe in 19, but for the domain.

Proposition 2.7. For any $A \in \mathcal{U}_{-\infty}(F)_{+}, \varphi(A)=\operatorname{Lim}_{\omega} \frac{\int_{K_{n}} \operatorname{tr}(a(x, x)) d x}{\operatorname{vol}\left(K_{n}\right)}$, which is Roe's definition in $[19$. 
Proof. Follows easily from the proof of Proposition 2.6.

Lemma 2.8. If $A \in \mathcal{J}_{0+}$ then

$$
\varphi(A)=\operatorname{Lim}_{\omega} \frac{\mu_{A}\left(K_{n}\left(r_{1}\right)\right)}{\operatorname{vol}\left(K_{n}\left(r_{2}\right)\right)}
$$

for any $r_{1}, r_{2} \in \mathbb{R}$.

Proof. Indeed, if $r_{1} \geq 0$, we get

$\operatorname{Lim}_{\omega} \frac{\mu_{A}\left(K_{n}\left(r_{1}\right)\right)}{\operatorname{vol}\left(K_{n}\left(r_{2}\right)\right)}=\operatorname{Lim}_{\omega}\left(\frac{\mu_{A}\left(K_{n}\right)}{\operatorname{vol}\left(K_{n}\right)}+\frac{\mu_{A}\left(K_{n}\left(r_{1}\right) \backslash K_{n}\right)}{\operatorname{vol}\left(K_{n}\right)}\right) \frac{\operatorname{vol}\left(K_{n}\right)}{\operatorname{vol}\left(K_{n}\left(r_{2}\right)\right)}=\varphi(A)$

whereas, if $r_{1}<0$, we get

$\operatorname{Lim}_{\omega} \frac{\mu_{A}\left(K_{n}\left(r_{1}\right)\right)}{\operatorname{vol}\left(K_{n}\left(r_{2}\right)\right)}=\operatorname{Lim}_{\omega}\left(\frac{\mu_{A}\left(K_{n}\right)}{\operatorname{vol}\left(K_{n}\right)}-\frac{\mu_{A}\left(K_{n} \backslash K_{n}\left(r_{1}\right)\right)}{\operatorname{vol}\left(K_{n}\right)}\right) \frac{\operatorname{vol}\left(K_{n}\right)}{\operatorname{vol}\left(K_{n}\left(r_{2}\right)\right)}=\varphi(A)$.

The algebra $\mathcal{A}$, being a $\mathrm{C}^{*}$-algebra, contains many unitary operators, and is indeed generated by them. The algebra $\mathcal{A}_{0}$ may not, but all unitaries in $\mathcal{A}$ may be approximated by elements in $\mathcal{A}_{0}$. Such approximants are $\delta$-unitaries, according to the following

Definition 2.9. An operator $U \in \mathcal{B}\left(L^{2}(F)\right)$ is called $\delta$-unitary, $\delta>0$, if $\left\|U^{*} U-1\right\|<\delta$, and $\left\|U U^{*}-1\right\|<\delta$.

Let us denote with $\mathcal{U}_{\delta}$ the set of $\delta$-unitaries in $\mathcal{A}_{0}$ and observe that, if $\delta<1$, $\mathcal{U}_{\delta}$ consists of invertible operators, and $U \in \mathcal{U}_{\delta}$ implies $U^{-1} \in \mathcal{U}_{\delta /(1-\delta)}$.

Proposition 2.10. The weight $\varphi$ is $\varepsilon$-invariant for $\delta$-unitaries in $\mathcal{A}_{0}$, namely, for any $\varepsilon \in(0,1)$, there is $\delta>0$ s.t., for any $U \in \mathcal{U}_{\delta}$, and $A \in \mathcal{A}_{+}$,

$$
(1-\varepsilon) \varphi(A) \leq \varphi\left(U A U^{*}\right) \leq(1+\varepsilon) \varphi(A) .
$$

Lemma 2.11. If $T \in \mathcal{J}_{0+}$, then $A T A^{*} \in \mathcal{J}_{0+}$ for all $A \in \mathcal{A}_{0}$.

Proof. First observe that for any Borel set $\Omega \subset M$ we have

$$
\begin{aligned}
\mu_{A T A^{*}}(\Omega) & =\operatorname{Tr}\left(E_{\Omega} A T A^{*} E_{\Omega}\right) \\
& =\operatorname{Tr}\left(E_{\Omega} A E_{\Omega\left(u_{A}\right)} T E_{\Omega\left(u_{A}\right)} A^{*} E_{\Omega}\right) \\
& \leq\left\|A^{*} E_{\Omega} A\right\| \operatorname{Tr}\left(E_{\Omega\left(u_{A}\right)} T E_{\Omega\left(u_{A}\right)}\right) \\
& \leq\|A\|^{2} \mu_{T}\left(\Omega\left(u_{A}\right)\right)
\end{aligned}
$$


so that

$$
\begin{aligned}
\frac{\mu_{A T A^{*}}\left(K_{n}\right)}{\operatorname{vol}\left(K_{n}\right)} & \leq\|A\|^{2} \frac{\mu_{T}\left(K_{n}\left(u_{A}\right)\right)}{\operatorname{vol}\left(K_{n}\right)} \\
& =\|A\|^{2} \frac{\mu_{T}\left(K_{n}\right)}{\operatorname{vol}\left(K_{n}\right)}+\|A\|^{2} \frac{\mu_{T}\left(K_{n}\left(u_{A}\right) \backslash K_{n}\right)}{\operatorname{vol}\left(K_{n}\right)}
\end{aligned}
$$

which is bounded. Now observe that, by Lemma 2.2 (iii), it follows

$$
\begin{aligned}
\frac{\mu_{A T A^{*}}\left(K_{n}\left(r_{1}\right) \backslash K_{n}\left(-r_{2}\right)\right)}{\operatorname{volK}_{n}} & \leq\|A\|^{2} \frac{\mu_{T}\left(\operatorname{Pen}^{+}\left(K_{n}\left(r_{1}\right) \backslash K_{n}\left(-r_{2}\right), u_{A}\right)\right)}{\operatorname{vol}_{n}} \\
& \leq\|A\|^{2} \frac{\mu_{T}\left(K_{n}\left(r_{1}+u_{A}+\varepsilon\right) \backslash K_{n}\left(-r_{2}-u_{A}-\varepsilon\right)\right)}{\operatorname{vol}_{n}} \rightarrow 0,
\end{aligned}
$$

the thesis follows.

Proof of Proposition 2.10. Assume $A \in \mathcal{J}_{0+} \cap \mathcal{A}_{+}$, then $U A U^{*} \in \mathcal{J}_{0+}$ and, by Lemma 2.8,

$$
\begin{aligned}
\varphi\left(U A U^{*}\right) & =\operatorname{Lim}_{\omega} \frac{\mu_{U A U^{*}}\left(K_{n}\right)}{\operatorname{vol}\left(K_{n}\right)} \\
& \leq\|U\|^{2} \operatorname{Lim}_{\omega}\left(\frac{\mu_{A}\left(K_{n}\left(u_{U}\right)\right)}{\operatorname{vol}\left(K_{n}\right)}\right) \\
& \leq(1+\delta) \varphi(A) .
\end{aligned}
$$

Choose now $\delta<\varepsilon / 2$, and $U \in \mathcal{U}_{\delta}$, so that $U^{-1} \in \mathcal{U}_{2 \delta}$, and $\varphi\left(U A U^{*}\right) \leq$ $(1+\delta) \varphi(A)<(1+\varepsilon) \varphi(A)$. Replacing $A$ with $U A U^{*}$, and $U$ with $U^{-1}$, we obtain

$$
\varphi(A) \leq\left\|U^{-1}\right\|^{2} \varphi\left(U A U^{*}\right) \leq(1+2 \delta) \varphi\left(U A U^{*}\right)<(1+\varepsilon) \varphi\left(U A U^{*}\right)
$$

and the thesis easily follows.

Assume now $A \in \mathcal{A}_{+} \backslash \mathcal{J}_{0+}$, then $\varphi(A)=+\infty=\varphi\left(U A U^{*}\right)$, because otherwise $U A U^{*} \in \mathcal{J}_{0+}$, so that $A=U^{-1}\left(U A U^{*}\right)\left(U^{-1}\right)^{*} \in \mathcal{J}_{0+}$, which is absurd.

Finally we observe that, from the proof of Lemma 2.11 the following is immediately obtained

Proposition 2.12. If $A \in \mathcal{A}_{0}$ and $\|A\| \leq 1$, then $\varphi\left(A T A^{*}\right) \leq \varphi(T)$, for any $T \in \mathcal{J}_{0+}$.

\section{A construction of semicontinuous traces on $\mathrm{C}^{*}$-algebras}

The purpose of this section is to show that the lower-semicontinuous semifinite regularisation of the functional $\left.\varphi\right|_{\mathcal{A}}$ of the previous section gives a trace, namely 
a unitarily invariant weight on $\mathcal{A}$. It turns out that this procedure can be applied to any weight $\tau_{0}$, on a unital $\mathrm{C}^{*}$-algebra $\mathcal{A}$, which is $\varepsilon$-invariant for $\delta$-unitaries of a dense ${ }^{*}$-subalgebra $\mathcal{A}_{0}$. The particular case of the functional $\left.\varphi\right|_{\mathcal{A}}$ is treated in the next section. First we observe that, with each weight on $\mathcal{A}$, namely a functional $\tau_{0}: \mathcal{A}_{+} \rightarrow[0, \infty]$, satisfying the property $\tau_{0}(\lambda A+B)=$ $\lambda \tau_{0}(A)+\tau_{0}(B), \lambda>0, A, B \in \mathcal{A}_{+}$, we may associate a (lower-)semicontinuous weight $\tau$ with the following procedure

$$
\tau(A):=\sup \left\{\psi(A): \psi \in \mathcal{A}_{+}^{*}, \psi \leq \tau_{0}\right\}
$$

Indeed, it is known that [5, 23.

$$
\tau(A) \equiv \sup _{\psi \in \mathcal{F}\left(\tau_{0}\right)} \psi(A)
$$

where $\mathcal{F}\left(\tau_{0}\right):=\left\{\psi \in \mathcal{A}_{+}^{*}: \exists \varepsilon>0,(1+\varepsilon) \psi<\tau_{0}\right\}$. Moreover the following holds

Theorem 3.1. 18 The set $\mathcal{F}\left(\tau_{0}\right)$ is directed, namely, for any $\psi_{1}, \psi_{2} \in \mathcal{F}\left(\tau_{0}\right)$, there is $\psi \in \mathcal{F}\left(\tau_{0}\right)$, s.t. $\psi_{1}, \psi_{2} \leq \psi$.

From this theorem easily follows

Corollary 3.2. Let $\tau_{0}$ be a weight on the $C^{*}$-algebra $\mathcal{A}$, and $\tau$ be defined as in (3.1). Then

(i) $\tau$ is a semicontinuous weight on $\mathcal{A}$

(ii) $\tau=\tau_{0}$ iff $\tau_{0}$ is semicontinuous.

(iii) The domain of $\tau$ contains the domain of $\tau_{0}$.

The weight $\tau$ will be called the semicontinuous regularization of $\tau_{0}$.

Proof. (i) From Theorem 3.1, $\tau(A)=\sup _{\psi \in \mathcal{F}\left(\tau_{0}\right)} \psi(A)=\lim _{\psi \in \mathcal{F}\left(\tau_{0}\right)} \psi(A)$, whence linearity and semicontinuity of $\tau$ easily follow.

(ii) is a well known result by Combes [5].

(iii) Immediately follows from the definition of $\tau$.

Proposition 3.3. Let $\tau_{0}$ be a weight on $\mathcal{A}$ which is $\varepsilon$-invariant by $\delta$-unitaries in $\mathcal{A}_{0}$ (as in Proposition 2.10). Then the associated semicontinuous weight $\tau$ satisfies the same property.

Proof. Fix $\varepsilon<1$ and choose $\delta \in(0,1 / 2)$, s.t. $U \in \mathcal{U}_{\delta}$ implies $\mid \tau_{0}\left(U A U^{*}\right)-$ $\tau_{0}(A) \mid<\varepsilon \tau_{0}(A), A \in \mathcal{A}_{+}$. Then, for any $U \in \mathcal{U}_{\delta / 2}$ and any $\psi \in \mathcal{A}_{+}^{*}, \psi \leq \tau_{0}$, we get

$$
\psi \circ \operatorname{adU}(A) \leq \tau_{0}\left(U A U^{*}\right) \leq(1+\varepsilon) \tau_{0}(A),
$$

for $A \in \mathcal{A}_{+}$, i.e. $(1+\varepsilon)^{-1} \psi \circ a d U \leq \tau_{0}$. Then

$$
\begin{aligned}
\tau\left(U A U^{*}\right) & =(1+\varepsilon) \sup _{\psi \leq \tau_{0}}(1+\varepsilon)^{-1} \psi \circ \operatorname{ad} U(A) \\
& \leq(1+\varepsilon) \sup _{\psi \leq \tau_{0}} \psi(A) \\
& =(1+\varepsilon) \tau(A)
\end{aligned}
$$


Since $U^{-1} \in \mathcal{U}_{\delta}$, replacing $U$ with $U^{-1}$ and $A$ with $U A U^{*}$, we get $\tau(A) \leq$ $(1+\varepsilon) \tau\left(U A U^{*}\right)$. Combining the last two inequalities, we get the result.

Theorem 3.4. The semicontinuous weight $\tau$ of Proposition 3.5 is a trace on $\mathcal{A}$, namely, setting $\mathcal{J}_{+}:=\left\{A \in \mathcal{A}_{+}: \tau(A)<\infty\right\}$, and extending $\tau$ to the linear span $\mathcal{J}$ of $\mathcal{J}_{+}$, we get

(i) $\mathcal{J}$ is an ideal in $\mathcal{A}$

(ii) $\tau(A B)=\tau(B A)$, for all $A \in \mathcal{J}, B \in \mathcal{A}$.

Proof. (i) Let us prove that $\mathcal{J}_{+}$is a unitary invariant face in $\mathcal{A}_{+}$, and it suffices to prove that $A \in \mathcal{J}_{+}$implies $U A U^{*} \in \mathcal{J}_{+}$, for all $U \in \mathcal{U}(\mathcal{A})$, the set of unitaries in $\mathcal{A}$. Suppose on the contrary that there is $U \in \mathcal{U}(\mathcal{A})$ s.t. $\tau\left(U A U^{*}\right)=\infty$. Then there is $\psi \in \mathcal{A}_{+}^{*}, \psi \leq \tau_{0}$, s.t. $\psi\left(U A U^{*}\right)>2 \tau(A)+2$. Then we choose $\delta<3$ s.t. $V \in \mathcal{U}_{\delta}$ implies $\tau\left(V A V^{*}\right) \leq 2 \tau(A)$, and an operator $U_{0} \in \mathcal{A}_{0}$ s.t. $\left\|U-U_{0}\right\|<\min \left\{\frac{\delta}{3}, \frac{1}{3\|A\|\|\psi\|}\right\}$. The inequalities

$$
\left\|U_{0} U_{0}^{*}-1\right\|=\left\|U^{*} U_{0} U_{0}^{*}-U^{*}\right\| \leq\left\|U^{*} U_{0}-1\right\|\left\|U_{0}^{*}\right\|+\left\|U_{0}^{*}-U^{*}\right\|<\delta
$$

and analogously for $\left\|U_{0}^{*} U_{0}-1\right\|<\delta$, show that $U_{0} \in \mathcal{U}_{\delta}$. Then, since $\mid \psi\left(U_{0} A U_{0}^{*}\right)-$ $\psi\left(U A U^{*}\right) \mid \leq 3\|\psi\|\|A\|\left\|U-U_{0}\right\|<1$, we get

$$
2 \tau(A) \geq \tau\left(U_{0} A U_{0}^{*}\right) \geq \psi\left(U_{0} A U_{0}^{*}\right) \geq \psi\left(U A U^{*}\right)-1 \geq 2 \tau(A)+1
$$

which is absurd.

(ii) We only have to show that $\tau$ is unitary invariant. Take $A \in \mathcal{J}_{+}, U \in \mathcal{U}(\mathcal{A})$. For any $\varepsilon>0$ we may find a $\psi \in \mathcal{A}_{+}^{*}, \psi \leq \tau_{0}$, s.t. $\psi\left(U A U^{*}\right)>\tau\left(U A U^{*}\right)-\varepsilon$, as, by $(i), \tau\left(U A U^{*}\right)$ is finite. Then, arguing as in the proof of $(i)$, we may find $U_{0} \in \mathcal{A}_{0}$, so close to $U$ that

$$
\begin{aligned}
& \left|\psi\left(U_{0} A U_{0}^{*}\right)-\psi\left(U A U^{*}\right)\right|<\varepsilon \\
& (1-\varepsilon) \tau(A) \leq \tau\left(U_{0} A U_{0}^{*}\right) \leq(1+\varepsilon) \tau(A) .
\end{aligned}
$$

Then

$$
\begin{aligned}
\tau(A) & \geq \frac{1}{1+\varepsilon} \tau\left(U_{0} A U_{0}^{*}\right) \geq \frac{1}{1+\varepsilon} \psi\left(U_{0} A U_{0}^{*}\right) \\
& \geq \frac{1}{1+\varepsilon}\left(\psi\left(U A U^{*}\right)-\varepsilon\right) \geq \frac{1}{1+\varepsilon}\left(\tau\left(U A U^{*}\right)-2 \varepsilon\right) .
\end{aligned}
$$

By the arbitrariness of $\varepsilon$ we get $\tau(A) \geq \tau\left(U A U^{*}\right)$. Replacing $A$ with $U A U^{*}$, we get the thesis.

The second regularization we need turns $\tau$ into a (lower semicontinuous) semifinite trace, namely guarantees that

$$
\tau(A)=\sup \left\{\tau(B): 0 \leq B \leq A, B \in \mathcal{J}_{+}\right\}
$$

for all $A \in \mathcal{A}_{+}$. In particular the semifinite regularization coincides with the original trace on the domain of the latter. This regularization is well known (see 
e.g. [7], Section 6), and amounts to represent $\mathcal{A}$ via the GNS representation $\pi$ induced by $\tau$, define a normal semifinite faithful trace $\operatorname{tr}$ on $\pi(\mathcal{A})^{\prime \prime}$, and finally pull it back on $\mathcal{A}$, that is $\operatorname{tr} \circ \pi$. It turns out that $\operatorname{tr} \circ \pi$ is (lower semicontinuous and) semifinite on $\mathcal{A}$, $\operatorname{tr} \circ \pi \leq \tau$, and $\operatorname{tr} \circ \pi(A)=\tau(A)$ for all $A \in \mathcal{J}_{+}$, that is $\operatorname{tr} \circ \pi$ is a semifinite extension of $\tau$, and $\operatorname{tr} \circ \pi=\tau$ iff $\tau$ is semifinite.

We still denote by $\tau$ its semifinite extension. As follows from the construction, semicontinuous semifinite traces are exactly those of the form $t r \circ \pi$, where $\pi$ is a tracial representation, and $t r$ is a n.s.f. trace on $\pi(\mathcal{A})^{\prime \prime}$.

\section{The semicontinuous trace on almost local op- erators and the Novikov-Shubin numbers}

Now we apply the regularization procedure described in the previous section to Roe's functional. Let us remark that the semicontinuous regularization of the weight $\left.\varphi\right|_{\mathcal{A}}$ is a trace in the sense of property (ii) of Theorem 3.4, which is stronger then the trace property in [19]. First we observe that $\left.\varphi\right|_{\mathcal{A}}$ is not semicontinuous.

Proposition 4.1. The set $\mathcal{N}_{0}:=\left\{T \in \mathcal{A}_{+}: \varphi(T)=0\right\}$ is not closed. In particular, there are operators $T \in \mathcal{A}_{+}$s.t. $\varphi(T)=1$ but $\tau(T)=0$ for any (lower-)semicontinuous trace $\tau$ dominated by $\left.\varphi\right|_{\mathcal{A}}$.

Proof. Recall from Lemma $1.2(i)$ that there are positive real functions $\beta_{1}, \beta_{2}$ s.t. $0<\beta_{1}(r) \leq V(x, r) \leq \beta_{2}(r)$, for all $x \in M, r>0$, and $\lim _{r \rightarrow 0} \beta_{2}(r)=0$. Therefore we can find a sequence $r_{n} \searrow 0$ s.t. $\sum_{n=1}^{\infty} \beta_{2}\left(r_{n}\right)<\infty$. Fix $o \in M$, and set $X_{n}:=\left\{\left(x_{1}, x_{2}\right) \in M \times M: n \leq \delta\left(x_{i}, o\right) \leq n+1, \delta\left(x_{1}, x_{2}\right) \leq r_{n}\right\}$, $Y_{n}:=\cup_{k=1}^{n} X_{k}, n \leq \infty$, and finally let $T_{n}$ be the integral operator whose kernel, a section of $\operatorname{End}(F)$ denoted $k_{n}$, is the characteristic function of $Y_{n}$. Since $k_{n}$ has compact support, if $n<\infty, \varphi\left(T_{n}\right)=0$. On the contrary, since $Y_{\infty}$ contains the diagonal of $M \times M$, clearly $\varphi\left(T_{\infty}\right)=1$. Finally, by Lemma 1.4,

$$
\begin{aligned}
\left\|T_{\infty}-T_{n}\right\| & \leq \sup _{x \in M} \sum_{k=n+1}^{\infty} \int_{M} \chi_{X_{k}}(x, y) d y \\
& \leq \sup _{x \in M} \sum_{k=n+1}^{\infty} V\left(x, r_{k}\right) \leq \sum_{k=n+1}^{\infty} \beta_{2}\left(r_{k}\right) \rightarrow 0 .
\end{aligned}
$$

This proves both the assertions.

Theorem 4.2. Let $M$ be an open manifold of $C^{\infty}$-bounded geometry, endowed with a regular exhaustion $\mathcal{K}$, and $F$ a finite dimensional hermitian vector bundle on $M$. Then the regularisation $\operatorname{Tr}_{\mathcal{K}}$ of $\varphi$ is a semicontinuous semifinite trace on the $C^{*}$-algebra $\mathcal{A}(F)$ of almost local operators on $L^{2}(F)$, which vanishes on compact operators. 
Proof. The first statement follows by the results of the previous section. Clearly $\varphi$, hence $T r_{\mathcal{K}}$, vanishes of finite rank operators. By semicontinuity, the kernel of $\operatorname{Tr}_{\mathcal{K}}$ is closed, which implies the thesis.

Finally we give a sufficient criterion for a positive operator $A \in \mathcal{A}$ to satisfy $\operatorname{Tr}_{\mathcal{K}}(A)=\varphi(A)$.

Proposition 4.3. Let $A \in \mathcal{J}_{0+}$ be an integral operator, whose kernel a $(x, y)$ is a section of End $(F)$ which is uniformly continuous in a neighborhood of the diagonal in $M \times M$, namely

$$
\forall \varepsilon>0, \exists \delta_{\varepsilon}>0: \delta(x, y)<\delta \Rightarrow|a(x, y)-a(x, x)|<\varepsilon .
$$

Then $\operatorname{Tr}_{\mathcal{K}}(A)=\varphi(A)$.

Proof. Consider first a family of integral operators $B_{\delta}$, with kernels, which are sections of $\operatorname{End}(F)$, given by

$$
b_{\delta}(x, y):=\frac{\beta_{1}(\delta)}{\beta_{2}(\delta)} \frac{\chi_{\Delta_{\delta}}(x, y)}{V(x, \delta)}
$$

where $\Delta_{\delta}:=\{(x, y) \in M \times M: \delta(x, y)<\delta\}$. Set $E_{n}$ for the multiplication operator by the characteristic function of $K_{n}$, and observe that

$$
\operatorname{Tr}\left(E_{n} B_{\delta} B_{\delta}^{*} E_{n}\right)=\frac{\beta_{1}(\delta)^{2}}{\beta_{2}(\delta)^{2}} \int_{K_{n}} \frac{d x}{V(x, \delta)} \leq \frac{\operatorname{vol}\left(K_{n}\right)}{\beta_{2}(\delta)}
$$

Therefore $\varphi\left(B_{\delta} B_{\delta}^{*}\right) \leq \beta_{2}(\delta)^{-1}$, hence $\psi_{\delta}:=\varphi\left(B_{\delta} \cdot B_{\delta}^{*}\right)$ is a positive functional on $\mathcal{A}$. Since $\sup _{x \in M} \int_{M} b_{\delta}(x, y) d y=\frac{\beta_{1}(\delta)}{\beta_{2}(\delta)} \leq 1$, and $\sup _{y \in M} \int_{M} b_{\delta}(x, y) d x \leq$ $\frac{\sup _{y \in M} V(y, \delta)}{\beta_{2}(\delta)} \leq 1$, Riesz-Thorin theorem implies $\left\|B_{\delta}\right\| \leq 1$, hence $\psi_{\delta} \leq\left.\varphi\right|_{\mathcal{A}}$ by Proposition 2.12. By definition of $T r_{\mathcal{K}}$, we have $\psi_{\delta} \leq T r_{\mathcal{K}}$.

Take now $A \in \mathcal{A}_{+}$satisfying (4.1), $\varepsilon>0, \delta=\delta_{\varepsilon}<\varepsilon$, and set $\beta(\delta):=\left(\frac{\beta_{1}(\delta)}{\beta_{2}(\delta)}\right)^{2}$. Then

$$
\begin{aligned}
& \left|\operatorname{Tr}\left(E_{n} B_{\delta} A B_{\delta}^{*} E_{n}\right)-\beta(\delta) \operatorname{Tr}\left(E_{n} A E_{n}\right)\right| \\
& \leq \int_{K_{n}} d x \int_{B(x, \delta) \times B(x, \delta)} b_{\delta}(x, y)|a(y, z)-a(x, x)| b_{\delta}(x, z) d y d z \\
& \leq 3 \varepsilon \int_{K_{n}} d x \int_{B(x, \delta) \times B(x, \delta)} b_{\delta}(x, y) b_{\delta}(x, z) d y d z \leq 3 \varepsilon \beta(\delta) \operatorname{vol}\left(K_{n}\right),
\end{aligned}
$$

hence $\left|\varphi(A)-\operatorname{Tr}_{\mathcal{K}}(A)\right| \leq\left|\varphi(A)-\psi_{\delta}(A)\right| \leq 3 \varepsilon \beta(\delta)+(1-\beta(\delta)) \varphi(A)$. The thesis follows by Lemma 1.2( $(i i)$.

Theorem 4.4. Let $M$ be an open manifold of $C^{\infty}$-bounded geometry, endowed with a regular exhaustion $\mathcal{K}, p \in\{0,1, \ldots, \operatorname{dim} M\}$ and $\operatorname{Tr}_{\mathcal{K}}$ the trace on 
$\mathcal{A}\left(\Lambda^{p} T^{*} M\right)$ given by Theorem 4.2. Then $\mathrm{e}^{-t \Delta_{p}}$ belongs to the domain of $\operatorname{Tr}_{\mathcal{K}}$, for any $t>0$, and

$$
\operatorname{Tr}_{\mathcal{K}}\left(\mathrm{e}^{-t \Delta_{p}}\right)=\operatorname{Lim}_{\omega} \frac{\int_{K_{n}} \operatorname{tr}\left(H_{p}(t, x, x)\right) d x}{\operatorname{vol}\left(K_{n}\right)},
$$

where $H_{p}$ is the heat kernel on $p$-forms.

Proof. By Remark 2.5 and Theorem 1.6 we have that $\mathrm{e}^{-t \Delta_{p}}$ belongs to $\partial_{0+} \cap \mathcal{A}$, hence, by Corollary 3.2, (iii), it belongs to the domain of $T r_{\mathcal{K}}$. The equality then follows by Propositions 1.3, 4.3, and 2.7.

We note that the previous result can be easily extended to functional calculi $f(\Delta)$ where $f$ has exponential decay.

We conclude this section showing that the above defined trace can be used to define the Novikov-Shubin numbers for open manifolds. Let $M$ be an open $n$-manifold with $\mathrm{C}^{\infty}$-bounded geometry possessing a regular exhaustion, and denote by $\mathcal{A}_{p} \equiv \mathcal{A}\left(\Lambda^{p} T^{*} M\right)$ the $\mathrm{C}^{*}$-algebra of almost local operators acting on $L^{2}\left(\Lambda^{p} T^{*} M\right)$. Let us define a positive measure $\mu_{p}$ on $[0, \infty)$ as

$$
\operatorname{Tr}_{\mathcal{K}}\left(f\left(\Delta_{p}\right)\right)=\int f(t) d \mu_{p}(t), \quad f \in C_{c}[0, \infty),
$$

and denote by $N_{p}(t):=\mu_{p}([0, t))$ the spectral density function of $\Delta$, and by $\vartheta_{p}$ the function $\vartheta_{p}(t):=\operatorname{Tr}_{\mathcal{K}}\left(e^{-t \Delta_{p}}\right)=\int_{0}^{\infty} \mathrm{e}^{-t \lambda} d \mu_{p}(\lambda)$. Then $\vartheta_{p}$ can be written as $\vartheta_{p}(t)=\int_{0}^{\infty} \mathrm{e}^{-t \lambda} d N_{p}(\lambda)$ so that, by a Tauberian theorem (10], Appendix), $\lim _{t \rightarrow 0} N_{p}(t)=\lim _{t \rightarrow \infty} \vartheta_{p}(t)$.

Definition 4.5. We define $b_{p} \equiv b_{p}(M, \mathcal{K}):=\lim _{t \rightarrow 0} N_{p}(t)=\lim _{t \rightarrow \infty} \vartheta_{p}(t)$ to be the $p$-th $\mathrm{L}^{2}$-Betti number of the open manifold $M$ endowed with the exhaustion $\mathcal{K}$. Let us now set $N_{p}^{0}(t):=N_{p}(t)-b_{p} \equiv \mu_{p}((0, t))$, and $\vartheta_{p}^{0}(t):=$ $\vartheta_{p}(t)-b_{p}=\int_{0}^{\infty} \mathrm{e}^{-t \lambda} d N_{p}^{0}(\lambda)$. The Novikov-Shubin numbers of $(M, \mathcal{K})$ are then defined as

$$
\begin{aligned}
& \alpha_{p} \equiv \alpha_{p}(M, \mathcal{K}):=2 \limsup _{t \rightarrow 0} \frac{\log N_{p}^{0}(t)}{\log t}, \\
& \underline{\alpha}_{p} \equiv \underline{\alpha}_{p}(M, \mathcal{K}):=2 \liminf _{t \rightarrow 0} \frac{\log N_{p}^{0}(t)}{\log t}, \\
& \alpha_{p}^{\prime} \equiv \alpha_{p}^{\prime}(M, \mathcal{K}):=2 \limsup _{t \rightarrow \infty} \frac{\log \vartheta_{p}^{0}(t)}{\log 1 / t}, \\
& \underline{\alpha}_{p}^{\prime} \equiv \underline{\alpha}_{p}^{\prime}(M, \mathcal{K}):=2 \liminf _{t \rightarrow \infty} \frac{\log \vartheta_{p}^{0}(t)}{\log 1 / t} .
\end{aligned}
$$

It follows from ([10], Appendix) that $\underline{\alpha}_{p}=\underline{\alpha}_{p}^{\prime} \leq \alpha_{p}^{\prime} \leq \alpha_{p}$, and $\alpha_{p}^{\prime}=\alpha_{p}$ if $\vartheta_{p}^{0}(t)=O\left(t^{-\delta}\right)$, for $t \rightarrow \infty$, or equivalently $N_{p}^{0}(t)=O\left(t^{\delta}\right)$, for $t \rightarrow 0$. Observe that $\mathrm{L}^{2}$-Betti numbers and Novikov-Shubin numbers depend on the limit procedure $\omega$ involved in the trace, and on the exhaustion $\mathcal{K}$. 
Remark 4.6. (i) $L^{2}$-Betti numbers for open manifolds have been defined by J. Roe (19]) as $\inf \left\{\phi\left(f\left(\Delta_{p}\right)\right): f \in C_{c}^{\infty}[0, \infty)_{+}, f(0)=1\right\}$, who showed in [20 their invariance under quasi-isometries. One can show easily that the two definitions coincide.

(ii) In [11] we used the semicontinuous semifinite trace constructed here to show that Novikov-Shubin numbers are asymptotic dimensions, and showed that they are invariant under quasi-isometries. Observe that the definition of the Novikov-Shubin numbers used there makes use of the noncommutative Riemann integration, which allows us to take the trace of many projections. Since the spectral projection $\chi_{[0, t)}\left(\Delta_{p}\right)$ is Riemann integrable for almost all $t$, the two definitions coincide.

(iii) Corollary 3.16 in [12] shows that the above definitions for $L^{2}$-Betti numbers and Novikov-Shubin numbers coincide with the classical ones in the case of amenable coverings, if one chooses the exhaustion given by the Følner condition.

In the case of coverings there is a well-known conjecture on the positivity of the $\alpha_{p}$ 's. A result by Varopoulos [24] shows that $\alpha_{0}$ is a positive integer, hence $\alpha_{0} \geq 1$. The following proposition extends this inequality to the case of open manifolds. Moreover $\alpha_{0}$ coincide with asymptotic dimension of $M$, cf. [13, for a suitable class of open manifolds, hence it can assume any value in $[1, \infty)$.

Proposition 4.7. Let $M$ be a complete non-compact Riemannian manifold of positive injectivity radius and Ricci curvature bounded from below. Then $\alpha_{0}(M, \mathcal{K})=\alpha_{0}^{\prime}(M, \mathcal{K}) \geq 1$ for any regular exhaustion $\mathcal{K}$.

Proof. Recall that, under the previous assumptions, Varopoulos [25] proved that the heat kernel on the diagonal has a uniform inverse-polynomial bound, more precisely, in the strongest form due to $\llbracket$, we have

$$
\sup _{x, y \in M} H_{0}(t, x, y) \leq C t^{-1 / 2}
$$

for a suitable constant $C$. Then, as

$$
\vartheta(t)=\tau\left(\mathrm{e}^{-t \Delta}\right)=\operatorname{Lim}_{\omega} \frac{\int_{B\left(o, n_{k}\right)} H_{0}(t, x, x) d v o l(x)}{V\left(o, n_{k}\right)} \leq C t^{-1 / 2},
$$

it follows from ([10], Appendix) that $\alpha_{0}=\alpha_{0}^{\prime}$, which concludes the proof.

\section{References}

[1] M. F. Atiyah. Elliptic operators, discrete groups and von Neumann algebras. Soc. Math. de France, Astérisque 32-33 (1976), 43-72.

[2] I. Buttig, J. Eichhorn. The heat kernel for p-forms on manifolds of bounded geometry. Acta Sci. Math., 55 (1991), 33-51.

[3] I. Chavel. Riemannian geometry - A modern introduction. Cambridge Univ. Press, Cambridge, 1993. 
[4] I. Chavel, E. A. Feldman. Modified isoperimetric constants, and large time heat diffusion in Riemannian manifolds. Duke J. Math., 64 (1991), 473499.

[5] F. Combes. Poids sur une $C^{*}$-algèbre. J. Math. pures et appl., 47 (1968), 57-100.

[6] A. Connes. Non Commutative Geometry. Academic Press, 1994.

[7] J. Dixmier. $C^{*}$-algebras. North-Holland Publ., Amsterdam, 1977.

[8] J. Dodziuk. De Rham-Hodge theory for $L^{2}$-cohomology of infinite coverings. Topology, 16 (1977), 157-165.

[9] M. Farber. Geometry of growth: approximation theorems for $L^{2}$ invariants. Math. Ann., 311 (1998), 335-375.

[10] M. Gromov, M. Shubin. Von Neumann spectra near zero. Geometric and Functional Analysis, 1 (1991), 375-404.

[11] D. Guido, T. Isola. Noncommutative Riemann integration and NovikovShubin invariants for open manifolds. J. Funct. Anal. 176 (2000) 115-152

[12] D. Guido, T. Isola. Singular traces, dimensions, and Novikov-Shubin invariants. Proceedings of the 17th OT Conference, Theta, Bucharest, 2000.

[13] D. Guido, T. Isola. An asymptotic dimension for metric spaces, and the 0-th Novikov-Shubin invariant. Preprint.

[14] E. Hewitt, K. Stromberg. Real and abstract analysis. Springer, New York, 1975.

[15] J. Lott. Heat kernels on covering spaces and topological invariants. J. Diff. Geom., 35 (1992), 471-510.

[16] C. C. Moore, C. Schochet. Global analysis on foliated spaces. Springer, New York, 1988.

[17] S. P. Novikov, M. A. Shubin. Morse theory and von Neumann $\mathrm{II}_{1}$ factors. Doklady Akad. Nauk SSSR, 289 (1986), 289-292.

[18] J. Quaegebeur, J. Verding. A construction for weights on $C^{*}$-algebras. Dual weights on $C^{*}$-crossed products. Preprint.

[19] J. Roe. An index theorem on open manifolds. I, II. J. Diff. Geom., 27 (1988), 87-136.

[20] J. Roe. On the quasi-isometry invariance of $L^{2}$ Betti numbers. Duke Math. J., 59 (1989), 765-783.

[21] J. Roe. Coarse cohomology and index theory on complete Riemannian manifolds. Memoirs A.M.S., 497 (1993), 1-90. 
[22] J. Roe. Index Theory, Coarse Geometry and Topology of Manifolds. CBMS Regional Conference Series in Mathematics, 90. American Mathematical Society, Providence, RI, 1996.

[23] S. Stratila. Modular theory in operator algebras, Abacus Press, Tunbridge Wells, England, 1981.

[24] N. T. Varopoulos. Random walks and Brownian motion on manifolds. Symposia Mathematica, XXIX (1987), 97-109.

[25] N. T. Varopoulos. Brownian motion and random walks on manifolds. Ann. Inst. Fourier, Grenoble, 34, 2, (1984), 243-269.

[26] J. Wolf. Essential self-adjointness for the Dirac operator and its square. Indiana Univ. Math. J. 22 (1972/73), 611-640. 